\title{
Delayed chemical meningitis after Chiari decompression with duraplasty: illustrative cases
}

\author{
Orgest Lajthia, MD, Mithun G. Sattur, MD, and Abhay K. Varma, MD \\ Department of Neurosurgery, Medical University of South Carolina, Charleston, South Carolina
}

BACKGROUND Dural reconstruction to achieve expansion duraplasty is important in suboccipital decompression for Chiari malformation type 1 (CM1). Although various dural substitutes are available, including synthetic collagen matrix grafts and dural xenografts, they have the potential to induce an inflammatory response. In this case series, the authors present their experience and discuss the incidence and possible mechanism of aseptic meningitis after the use of bovine collagen matrix graft as a dural substitute in patients with CM1 after suboccipital decompression.

OBSERVATIONS Three consecutive adult female patients who underwent suboccipital decompression at a single institution by a single neurosurgeon were retrospectively reviewed. They all presented with signs of aseptic meningitis in a delayed fashion, responded well to steroid administration, but had recurrence of their symptoms. Bovine collagen dural substitutes are resorbed in a process that induces an inflammatory response manifesting with signs of aseptic meningitis and is only alleviated with removal of the dural substitute.

LESSONS DuraMatrix Suturable, a dural xenograft derived from bovine dermis, though a viable choice for dural repair, is a potential cause of chemical meningitis after duraplasty in Chiari decompression surgery. In patients presenting with delayed and persistent aseptic meningitis after intervention, removal of this dural substitute led to improved symptomatology.

https://thejns.org/doi/abs/10.3171/CASE21331

KEYWORDS aseptic meningitis; chemical meningitis; dural grafts; duraplasty; Chiari malformation; suboccipital decompression

Dural reconstruction to achieve expansion duraplasty is an important component of surgery for Chiari malformation type 1 (CM1). ${ }^{1}$ Apart from autologous tissue such as pericranium, temporalis fascia, or fascia lata, a number of dural substitutes are available. These include synthetic collagen matrix grafts and dural xenografts. Although dural substitutes are convenient to use, they have the potential to induce an inflammatory response that may contribute to postoperative morbidity. ${ }^{2}$ We present three cases of aseptic meningitis after the use of DuraMatrix Suturable (Stryker), a bovine collagen matrix graft used as a dural substitute in symptomatic adult patients undergoing suboccipital decompression for CM1.

\section{Illustrative Cases}

Case 1

A 28-year-old female presented with occipital/suboccipital headaches and left facial and upper extremity numbness with worsening symptoms over the past 5 months. Brain magnetic resonance imaging (MRI) demonstrated 5-mm cerebellar tonsillar herniation with diminished but not obstructed cerebrospinal fluid (CSF) flow in the foramen magnum, whereas cervical spine MRI revealed a prominent central spinal canal from C6 to T1. The patient underwent surgery with an uneventful recovery and was discharged home on postoperative day 5 . She presented on postoperative day 12 with worsening headaches and neck pain. Her examination was notable for meningismus and a pseudomeningocele over the surgical site. MRI showed a pseudomeningocele at the surgical site without ventriculomegaly. CSF obtained through a lumbar puncture examination revealed lymphocytosis with a negative Gram stain and culture. The patient had a dramatic response to oral steroids and was discharged on a steroid taper. She presented again on postoperative day 48 with similar complaints. The pseudomeningocele appeared larger and more tense. The finding of repeat imaging was unchanged. CSF examination was notable for lymphocytosis and low CSF glucose.

ABBREVIATIONS CM1 = Chiari malformation type $1 ; \mathrm{CSF}=$ cerebrospinal fluid; $\mathrm{MRI}=$ magnetic resonance imaging INCLUDE WHEN CITING Published November 8, 2021; DOI: 10.3171/CASE21331.

SUBMITTED June 14, 2021. ACCEPTED September 16, 2021.

(c) 2021 The authors, CC BY-NC-ND 4.0 (http://creativecommons.org/licenses/by-nc-nd/4.0/). 
The Gram stain and culture results were negative. Her symptoms resolved again with oral steroids. She underwent placement of a lumbar-peritoneal shunt and removal of the suturable DuraMatrix graft (Stryker). Duraplasty was performed with autologous pericranium. She was discharged 1 week later with complete resolution of her symptoms. She was successfully tapered off steroids over the next few days. At her last follow-up at 70 weeks, she reported complete resolution of headache, pseudomeningocele, and original complaints related to $\mathrm{CM} 1$.

\section{Case 2}

A 43-year-old female patient presented with an 8-month history of worsening occipital headaches, neck pain, vomiting, upper extremity weakness, intermittent blurring of vision, dysphonia, and significant dysphagia causing weight loss. MRI revealed cerebellar tonsil herniation of $1.8 \mathrm{~cm}$ with significant brainstem and upper cervical spinal cord compression. The patient underwent surgery with subsequent improvement of her visual symptoms and upper extremity weakness. Her dysphagia persisted in the postoperative period, necessitating the placement of a gastrostomy tube. She was discharged on postoperative day 9. Her dysphagia and dysphonia improved, but she presented on postoperative day 39 with worsening headaches, neck pain, nausea/vomiting, meningismus, and a progressively enlarging pseudomeningocele at the surgical site. The imaging finding was unremarkable, except for a pseudomeningocele at the surgical site, and CSF examination demonstrated lymphocytosis with a negative Gram stain and culture result. Her symptoms markedly improved with oral steroids but recurred after completion of the steroid course. Repeat imaging at this time demonstrated a pseudomeningocele and hydrocephalus. Following ventriculoperitoneal shunt placement, the suturable DuraMatrix (Stryker) was removed, replaced with onlay DuraMatrix (Stryker), and reinforced with Tisseel (Baxter Healthcare). The patient's symptoms resolved after the procedure, and her gastrostomy tube was removed 12 weeks later. At 55-week follow-up, she reported complete resolution of her dysphagia, dysphonia, headaches, and motor weakness.

\section{Case 3}

A 47-year-old female presented with a long history of suboccipital headaches with associated dizziness and intermittent upper extremity numbness. Her cervical spine MRI demonstrated 7-mm tonsillar descent. She underwent an uneventful surgery and was discharged home on postoperative day 4 . The patient returned on postoperative day 12 with symptoms of meningitis. Imaging was unrevealing, and CSF examination revealed increased proteins, lymphocytosis, and eosinophilia (not mirrored by hematological eosinophilia) with a negative Gram stain and culture finding. She had a dramatic response to oral steroids but presented again with similar symptoms on postoperative day 23 after steroid taper. She was taken to the operating room, where she underwent placement of a temporary lumbar drain, removal of suturable DuraMatrix (Stryker), and replacement with onlay DuraMatrix (Stryker) and reinforced with Tisseel (Baxter Healthcare). Follow-up at 103 weeks revealed excellent index symptom improvement.

\section{Methods}

Data on 3 consecutive adult female patients who underwent suboccipital decompression for CM1 between February 1, 2018, and May 1, 2018, at the Medical University of South Carolina by the senior neurosurgeon were reviewed. Short- and intermediate-term complications, surgical technique, and type of dural graft used were noted. The surgical characteristics of our cohort are provided in Table 1.

\section{Operative Technique}

Standard suboccipital craniectomy measuring approximately 4-5 $\mathrm{cm} \times 3 \mathrm{~cm}$ along with $\mathrm{C} 1$ laminectomy was performed to accomplish foramen magnum decompression. The dura was opened in a Y-shaped fashion with the junction of horizontal and vertical limbs above the marginal sinus but sparing arachnoid. The arachnoid was then opened, and subpial decompression of the herniated tonsil was performed with an ultrasonic aspirator bilaterally. Watertight duraplasty was then performed with suturable DuraMatrix (Stryker) and running 5-0 or 6-0 Prolene sutures and reinforced with Tisseel (Baxter Healthcare) along the suture line. Suturable DuraMatrix (Stryker) was hydrated as per the manufacturer's instructions before implantation. The overlying fascia was closed in a watertight fashion with 2-0 Vicryl, and the skin layer was closed with running 2-0 nylon. All patients underwent lumbar drain insertion before positioning for surgery, and drainage was maintained for 3 to 5 days in the postoperative period.

\section{Discussion}

Options for duraplasty in the surgical management of Chiari malformation include autograft, allograft, xenograft, and synthetic nonbiological materials. The ideal dural graft should restore continuity of the dura mater, thus preventing CSF leaks, minimizing infection, and not initiating inflammatory reactions. Although the autograft meets most of these criteria, it may not be feasible in every case due to factors such as the need for a more extensive or separate incision, pain from graft harvest, nonavailability from radiation or prior surgery, and prolonged operative time.

One of the options is DuraMatrix Suturable (Stryker), a bovine dermis-derived xenograft with thickness similar to that of the native dura and a high suture retention strength that enables it to be sutured to surrounding tissue with minimal tear and that is eventually resorbed and replaced by new collagen. ${ }^{3}$ The process of resorption involves an inflammatory response from the host. ${ }^{4}$ Histopathological analysis of bovine collagen dural substitute at 4 weeks postoperatively corroborates the idea of local inflammatory and tissue-level responses elicited at the site of implantation. MacEwan et al. $^{2}$ demonstrated that xenograft material derived from bovine

TABLE 1. Surgical characteristics

\begin{tabular}{lccc}
\hline \multicolumn{1}{c}{ Variable } & Case 1 & Case 2 & Case 3 \\
\hline Dural graft & $\begin{array}{c}\text { Suturable } \\
\text { DuraMatrix }\end{array}$ & $\begin{array}{c}\text { Suturable } \\
\text { DuraMatrix }\end{array}$ & $\begin{array}{c}\text { Suturable } \\
\text { DuraMatrix }\end{array}$ \\
\hline Dural sutures & 6-0 Prolene & 6-0 Prolene & 5-0 Prolene \\
\hline Tisseel & Yes & Yes & Yes \\
\hline Length of stay, days & 5 & 9 & 4 \\
\hline Revision surgery, days & 51 & 51 & 35 \\
\hline Dural graft & $\begin{array}{c}\text { Periosteal } \\
\text { graft }\end{array}$ & $\begin{array}{c}\text { Onlay } \\
\text { DuraMatrix }\end{array}$ & $\begin{array}{c}\text { Onlay } \\
\text { DuraMatrix }\end{array}$ \\
\hline Dural sutures & 6-0 Prolene & None & None \\
\hline Shunt & Lumboperitoneal & VPS & None \\
\hline
\end{tabular}

VPS = ventriculoperitoneal shunt. 
sources exhibited fibrosis and fibrous capsule formation, increased cortical adhesions, reduced neoduralization, and increased recruitment of inflammatory cells compared with nonbiologic nanofabricated implants. Induction of an inflammatory response by bovine collagen grafts is not unique to dural substitutes and has been observed and described in other applications of dermal collagen matrices. ${ }^{5}$ Potentially, a persistent inflammatory response can induce generalized meningeal irritation, presenting with meningitis-like symptoms and signs of aseptic meningitis. "Aseptic meningitis" refers to meningeal inflammation not related to an infectious process with a wide range of etiological factors, including systemic diseases with meningeal involvement, neoplastic meningitis, drug-induced, immunogenic foreign body, and neurosurgical procedures. Presenting symptoms generally include headache, neck pain, nausea, and vomiting, with or without fever. Typical CSF findings demonstrate pleocytosis with lymphocytic predominance (or atypical cells in malignant meningitis), hypoglycorrhachia, elevated protein, and negative cultures.

All 3 patients in the our series presented with headache and meningismus without fever. CSF analysis revealed a lymphocytic predominance in every patient, with eosinophilia in 1 patient (Table 2). Gram stain and culture findings were negative in all 3 . Two patients presented on postoperative day 12, and 1 patient presented on postoperative day 39 , though the last patient had been experiencing symptoms for a few days before presentation. In all 3 patients, symptoms improved dramatically with a pulse dose of steroids, only to recur after the steroids were tapered off and to resolve completely only after graft removal. It is likely that the dural substitute used caused a local inflammatory reaction with aseptic meningitis.

The other potential immunogenic material used in these patients was Tisseel (Baxter Healthcare), a human fibrin sealant. However, Tisseel was also used in the revision surgery, wherein DuraMatrix Suturable (Stryker) was replaced with other dural substitutes, after which aseptic meningitis resolved. Hence, it is unlikely to be the cause of aseptic meningitis in this series.

Complications related to the use of a dural substitute in the surgical management of Chiari malformation has been reported previously. Parker et al. ${ }^{4}$ reported an overall complication rate of $21.1 \%$ with the use of dural substitutes with and without the addition of fibrin glue after duraplasty for CM1. The complications included aseptic meningitis, symptomatic pseudomeningocele, and CSF leak. Their report did not differentiate between dural substitute and fibrin glue as the source of aseptic meningitis. In a recent meta-analysis

\section{TABLE 2. CSF findings}

\begin{tabular}{lrrr}
\hline \multicolumn{1}{c}{ Variable } & Case 1 & Case 2 & Case 3 \\
\hline Red blood cells, $\mathrm{mm}^{3}$ & 150 & 83 & 207 \\
\hline White blood cells, $\mathrm{mm}^{3}$ & 215 & 11 & 605 \\
\hline Glucose, $\mathrm{mg} / \mathrm{dl}$ & 49 & 43 & 58 \\
\hline Protein, $\mathrm{mg} / \mathrm{dl}$ & 53 & 180 & 138 \\
\hline Nucleated cells, $\mathrm{mm}^{3}$ & 250 & 12 & 703 \\
\hline Neutrophils, \% & 3 & 6 & 1 \\
\hline Lymphocytes, \% & 78 & 85 & 35 \\
\hline Eosinophils, \% & 5 & 0 & 50 \\
\hline Monocytes/macrophages, \% & 14 & 9 & 14 \\
\hline Culture & Negative & Negative & Negative \\
\hline
\end{tabular}

looking at all the current evidence surrounding outcomes for various dural graft materials in posterior fossa decompression with duraplasty, it was found that complication rates were similar among patients whose dural grafts for CM1 were performed with autograft, synthetic graft, bovine pericardium, collagen-based graft, or allograft. ${ }^{6}$ Not surprisingly, autografts most frequently had the lowest rate of complications and were associated with significantly lower rates of pseudomeningocele than other dural substitutes. ${ }^{6}$

The complications seen in our cohort were confined to a 3month period, and this temporal relationship could suggest that there was something inherently unusual about the batch of dural substitutes used. Although objective data behind this theory are lacking, other factors surrounding the procedure, such as the surgeon and surgical technique, remained the same.

\section{Observations}

Suturable DuraMatrix, a dural xenograft derived from bovine dermis, though a viable choice for dural repair, is a potential cause of chemical (aseptic) meningitis after duraplasty in Chiari decompression surgery. CSF findings are notable for lymphocytosis with or without eosinophilia. In patients with delayed and persistent aseptic meningitis after surgery, removal of this dural substitute may be indicated.

These observations are important because duraplasty with a dural substitute is an essential step in Chiari decompression, and surgeons should be informed of the possible complications arising from the use of certain products. Observations related to the use of suturable DuraMatrix for duraplasty in patients with CM1 has not been previously reported.

\section{Lessons}

Aseptic meningitis is a rare but known complication associated with surgical management of Chiari malformation, and it is important to recognize the potential sources, including the dural graft. The limitation of this report is that the explanted dural graft was not submitted for histological examination.

\section{References}

1. Costa BS, Cavalcanti-Mendes G de A, Abreu MS, Sousa AA. Clinical experience with a novel bovine collagen dura mater substitute. Arq Neuropsiquiatr. 2011;69(2A):217-220.

2. MacEwan MR, Kovacs T, Osbun J, Ray WZ. Comparative analysis of a fully-synthetic nanofabricated dura substitute and bovine collagen dura substitute in a large animal model of dural repair. Interdiscip Neurosurg. 2018;13:145-150.

3. Abla AA, Link T, Fusco D, Wilson DA, Sonntag VKH. Comparison of dural grafts in Chiari decompression surgery: Review of the literature. J Craniovertebr Junction Spine. 2010;1(1):29-37.

4. Parker SR, Harris P, Cummings TJ, George T, Fuchs H, Grant G. Complications following decompression of Chiari malformation Type I in children: dural graft or sealant? J Neurosurg Pediatr. 2011;8(2):177-183.

5. Broderick G, McIntyre J, Noury M, et al. Dermal collagen matrices for ventral hernia repair: comparative analysis in a rat model. Hernia. 2012;16(3):333-343.

6. Yahanda AT, Simon LE, Limbrick DD. Outcomes for various dural graft materials after posterior fossa decompression with duraplasty for Chiari malformation type I: a systematic review and meta-analysis. J Neurosurg. Published online April 9, 2021. doi: 10.3171/2020.9.JNS202641. 


\section{Disclosures}

The authors report no conflict of interest concerning the materials or methods used in this study or the findings specified in this paper.

\section{Author Contributions}

Conception and design: all authors. Acquisition of data: Lajthia, Varma Analysis and interpretation of data: all authors. Drafting the article: all authors. Critically revising the article: all authors. Reviewed submitted version of manuscript: Lajthia, Varma. Approved the final version of the manuscript on behalf of all authors: Lajthia. Statistical analysis: Lajthia. Administrative/technical/material support: Lajthia, Varma. Study supervision: Lajthia, Varma.

\section{Correspondence}

Orgest Lajthia: Medical University of South Carolina, Charleston, SC. lajthia@musc.edu. 